\title{
OBSERVATIONS OF 1E 1740.7-2942 WITH ROSAT AND THE VLA ${ }^{1}$
}

\author{
William A. Heindl, Thomas A. Prince, and John M. Grunsfeld 2 \\ Division of Physics, Mathematics, and Astronomy, California Institute of Technology, \\ M.S. 220-47, Pasadena, CA 91125 \\ Received 1993 December 13; accepted 1994 February 1
}

\begin{abstract}
We have observed the Galactic black hole candidate 1E 1740.7-2942 in X-rays with both the ROSAT HRI and PSPC and at 1.5 and $4.9 \mathrm{GHz}$ with the VLA. From the HRI observation we derive a position for 1E $1740.7-2942$ of right ascension $=17^{\mathrm{h}} 43^{\mathrm{m}} 54.9$, declination $=-29^{\circ} 44^{\prime} 45^{\prime \prime} .3(\mathrm{~J} 2000)$, with a $90 \%$ confidence error circle of radius 8.5. Thermal bremsstrahlung fits to the PSPC data yield a column density of $1.12_{-0.18}^{+1.51}$ $\times 10^{23} \mathrm{~cm}^{-2}$, consistent with earlier X-ray measurements. The VLA observation of $4.9 \mathrm{GHz}$ revealed two sources. Source A, which is the core of a double aligned radio jet source (Mirabel et al. 1992), lies within the ROSAT error circle, further confirming its identification with 1E 1740.7-2942.
\end{abstract}

Subject headings: black hole physics - radiation mechanisms: nonthermal - radio continuum: stars stars: individual (1E 1740.7-2942) - X-rays: stars

\section{INTRODUCTION}

Since 1985, observations in the hard X-ray and soft $\gamma$-ray energy bands have shown $1 \mathrm{E}$ 1740.7-2942 to be the dominant source in the central few degrees of the Galactic center region (Skinner et al. 1987; Cook et al. 1991). This fact and the observation by SIGMA (Bouchet et al. 1991) of a transient hard excess in the spectrum suggestive of positron annihilation motivated a campaign of multiwavelength observations aimed at discovering the nature of this unusual source. The search for counterparts has been carried out at radio (Prince et al. 1991b; Mirabel et al. 1992, 1993; Gray, Cram, \& Ekers 1992), millimeter (Mirabel et al. 1991; Bally \& Leventhal 1991), infrared (Prince et al. 1991b; Mirabel \& Duc 1992; Djorgovski, Thompson, \& Mazzarella 1992), and optical (Skinner et al. 1991; Mereghetti et al. 1992; Leahy, Langill, \& Kwok 1992; Bignami, Caraveo, \& Mereghetti 1993) wavelengths. The radio observations have shown that the compact central core of a double radio jet lies within the X-ray error circle, and the millimeter observations reveal a line of sight alignment with a dense molecular cloud. If this molecular cloud is associated with 1E 1740.7-2942, it might provide a source of material for accretion as well as a medium for the slowing and annihilation of positrons present in the radio jets (Mirabel et al. 1991). It is therefore important to determine whether 1E 1740.7-2942 lies within the molecular cloud. The X-ray spectrum is a sensitive diagnostic of the source column depth, $N_{\mathrm{H}}$, and so can shed light on the relationship of $1 \mathrm{E} 1740.7-2942$ and the molecular cloud. Knowledge of the column depth is also important to the interpretation of optical and infrared data, as the level of extinction limits the sensitivity of counterpart searches at these wavelengths.

We report here the results of observations of $1 \mathrm{E}$ $1740.7-2942$ at X-ray wavelengths using the high-resolution

\footnotetext{
${ }^{1}$ The VLA is a facility of the National Astronomy Observatory which is operated by Associated Universities, Inc., under contract with the National Science Foundation.

${ }^{2}$ Current address: NASA Johnson Space Center, Code CB, Houston, TX 77058 .
}

imager (HRI) and position-sensitive proportional counter (PSPC) on ROSAT and at 1.5 and $4.9 \mathrm{GHz}$ using the Very Large Array (VLA) in Socorro, New Mexico. Preliminary results of the HRI and VLA observations have been reported in Prince et al. (1991a) and Prince et al. (1991b), respectively.

\section{OBSERVATIONS}

The HRI observation was performed during the period 1991 March 20-24. It incorporates 11 observation intervals of between 850 and $2500 \mathrm{~s}$ duration for a total live time of $19,900 \mathrm{~s}$. While 1E $1740.7-2942$ apparently remained in its normal state throughout 1990, observations by SIGMA (Mandrou et al. 1994) in 1991 February and March showed that 1E 1740.7-2942 entered a low state (hard X-ray luminosity $\sim 0.25$ of normal) prior to our HRI observation.

The PSPC observation was split over two observing periods - the first between 1992 September 28 and October 4 (hereafter PSPC-I) and the second between 1993 March 23 and 28 (hereafter PSPC-II). PSPC-I encompassed 12 observation intervals for a total livetime of $15,160 \mathrm{~s}$, and PSPC-II comprised seven observation intervals with a total livetime of 13,070 s. SIGMA observations prior to PSPC-I and spanning PSPC-II give an indication of the likely state of $1 \mathrm{E}$ 1740.7-2942 during the ROSAT pointings. In 1992 September, 1E 1740.7-2942 was seen to be in its normal state. By 1993 March it entered the "subluminous" state (Churazov et al. 1994) described by Cordier et al. (1993).

We observed the region of the Einstein IPC 1E 1740.7-2942 error circle (see Fig. 1) with the VLA on 1989 March 2. The observations were made at 1.5 and $4.9 \mathrm{GHz}$ for a duration of $4.5 \mathrm{hr}$ each. The array was in the $\mathrm{A} / \mathrm{B}$ configuration which provides a good point spread function for southern sources (Bridle 1986).

\section{ANALYSIS AND RESULTS}

\subsection{HRI Error Circle}

The HRI observation revealed three sources near the center of the field of view (FOV) (see Figs. 2 and 3 [Pl. 34]). Only source 1 is consistent in location with previous error circles for 


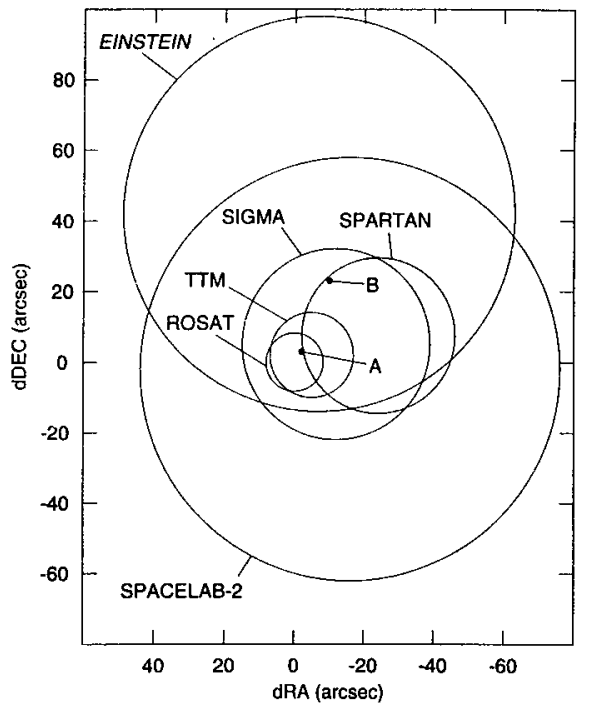

FIG. 1.-X-ray and hard X-ray error circles for 1E 1740.7-2942. Also shown are the positions of the VLA sources $A$ and $B$. Offsets are relative to the HRI X-ray position: R.A. $=17^{\mathrm{h}} 43^{\mathrm{m}} 54^{\mathrm{s}} 9$, Decl. $=-29^{\circ} 44^{\prime} 45^{\prime \prime}, 3$ (J2000). References: Einstein (Hertz \& Grindlay 1984); Spacelab 2 (Skinner et al. 1987); SIGMA (Cordier et al. 1993); Spartan-1 (Kawai et al. 1988); TTM (Skinner et al. 1991).

1E 1740.7-2942 (Hertz \& Grindlay 1984; Skinner et al. 1987, 1991; Kawai et al. 1988). In order to derive an HRI error circle, we applied corrections to the nominal image coordinates based on the HRI boresight offsets of Kürster \& Hasinger (1992), who give errors in the HRI positions of 23 sources with known accurate locations. Using their data and discarding two sources with anomalously large errors, we found the average offsets for the remaining 21 sources. We then plotted the integral distribution of radial offsets in order to estimate the $90 \%$ confidence radius for any detected source. These boresight corrections dominate the final position uncertainty, resulting in a $90 \%$ confidence error circle of radius 8.5 . The best-fit coordi-

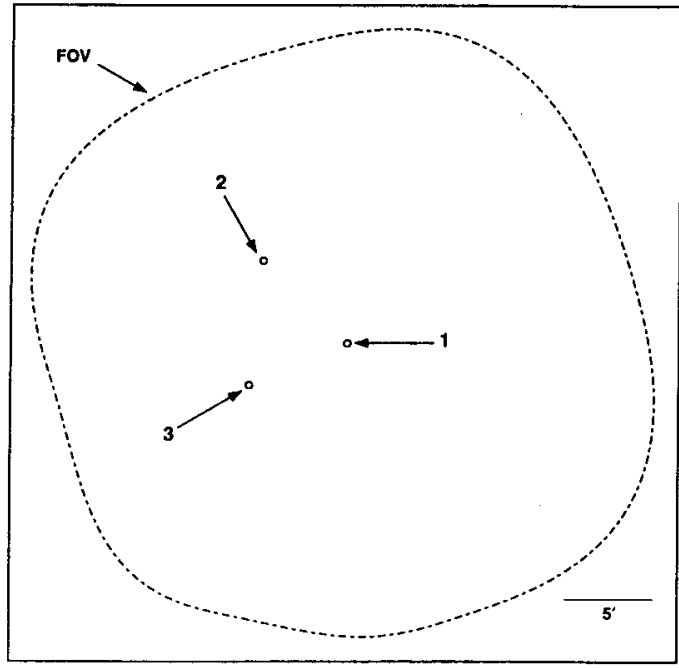

FIG. 2.-Diagram of the ROSAT HRI image of the 1E 1740.7-2942 field obtained during 1991 March 20-24. The actual image appears in Fig. 3. Three sources were detected near the center of the field. Only source 1 is consistent with previous X-ray error circles for 1E 1740.7-2942. North is up, and east is to the left. nates (epoch $\mathrm{J} 2000.0)$ to source $1(=1 \mathrm{E} 1740.7-2942)$, are right ascension $=17^{\mathrm{h}} 43^{\mathrm{m}} 54^{\mathrm{s}} .9$ and declination $=-29^{\circ} 44^{\prime} 45^{\prime \prime} .3$.

\subsection{Spectrum}

We analyzed the PSPC observations assuming both powerlaw and thermal bremsstrahlung models. Model parameters were estimated using the likelihood ratio method (Cash 1979). PSPC-I and PSPC-II were treated individually and also summed to form a single measurement. Table 1 summarizes the results of the spectral fits. Because the nominal PSPC energy range extends only to $2.5 \mathrm{keV}$, the data do not place an upper bound on the source temperature, and no physical constraints on a power-law index are obtained. Under the bremsstrahlung model, however, only a relatively narrow range of column densities are allowed for temperatures between 0.1 and $100 \mathrm{keV}$. In addition, models with temperatures below $\sim 0.5$ $\mathrm{keV}$ resulted in significantly worse fits than temperatures above $\sim 1 \mathrm{keV}$. For these reasons, the thermal bremsstrahlung column densities and fluxes in Table 1 were calculated assuming $k T=14.3 \mathrm{keV}$, while the column densities for the power-law model assumed a photon index of 2 , both corresponding to the best-fit values from the Spartan-1 measurement (Kawai et al. 1988). As a check on our spectral fits, we used the instrument response and spectral modeling software contained in the ROSAT Mission Information and Planning System (MIPS) to predict count rates for the best-fit column depth and normalization, based on the thermal bremsstrahlung spectra in Table 1 . The predicted rates were statistically consistent with the measured values. Figure 4 shows the measured source count spectrum together with a folded thermal bremsstrahlung spectrum $\left(k T=14.3 \mathrm{keV}, N_{\mathrm{H}}=1.12 \times 10^{23}\right.$ $\mathrm{cm}^{-2}$ ). We note that images produced from the pulse invariant energy channels greater than 256 (the limit of the current PSPC response matrix) show significant counts from 1E 1740.7-2942 and should provide improved fits when the response matrix is extended.

\subsection{Other Sources}

In addition to the HRI sources 2 and 3 , several sources were detected in the PSPC. Figures 5 and 6 (Plate 35) show the central region of the PSPC image for the entire observation including both PSPC-I and PSPC-II. 1E 1740.7-2942 and the well-known source A1742-294 (=1E 1742.9-2929) (van

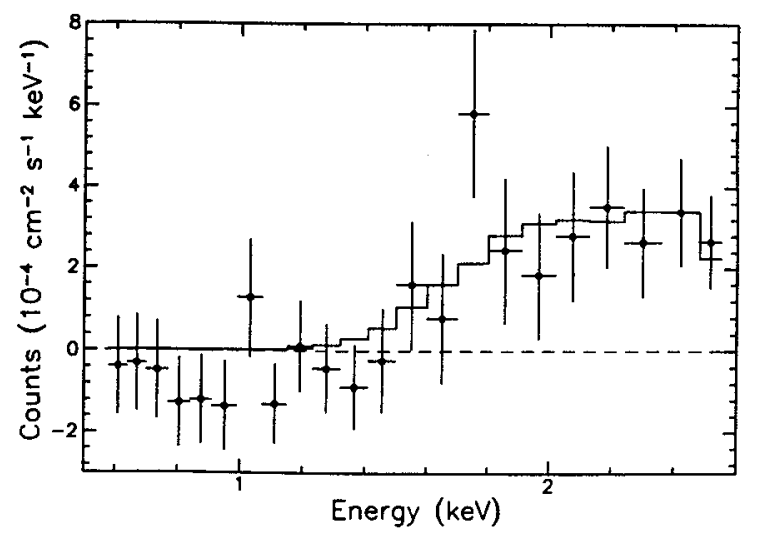

FIG. 4.-Background subtracted PSPC count spectrum of 1E 1740.7-2942 with folded thermal bremsstrahlung model. The model parameters are $k T=14.3 \mathrm{keV}, N_{\mathrm{H}}=1.12 \times 10^{23} \mathrm{~cm}^{-2}$. 
TABLE 1

SPECTRAL Fits TO THE ROSAT OBSERVATIONS

\begin{tabular}{|c|c|c|c|c|}
\hline Parameter & $\begin{array}{l}1990 \mathrm{Mar} \\
\text { HRI }\end{array}$ & $\begin{array}{l}1992 \text { Sep-Oct } \\
\text { PSPC-I }\end{array}$ & $\begin{array}{l}1993 \text { Mar } \\
\text { PSPC-II }\end{array}$ & $\begin{array}{l}\text { Combined } \\
\text { PSPC }\end{array}$ \\
\hline $\begin{array}{l}\text { Rate }\left(10^{-3} \mathrm{~s}^{-1}\right) \ldots \ldots \ldots \ldots \ldots \ldots \ldots \ldots \ldots \ldots \ldots \ldots \ldots \ldots \\
\text { Thermal Bremsstrahlung: }\end{array}$ & $1.9 \pm 0.3$ & $3.3 \pm 0.7$ & $2.0 \pm 0.7$ & $2.7 \pm 0.5$ \\
\hline 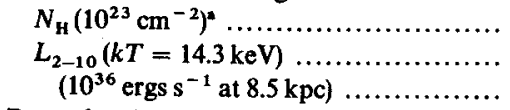 & $\sim 3.9^{\mathrm{b}}$ & $\begin{array}{l}1.26_{-0.21}^{+1.72} \\
2.6_{-1.4}^{+3.1}\end{array}$ & $\begin{array}{l}0.92_{-0.20}^{+1.58} \\
0.44_{-0.27}^{+0.57}\end{array}$ & $\begin{array}{l}1.12_{-0.18}^{+1.51} \\
1.3_{-0.6}^{+1.2}\end{array}$ \\
\hline 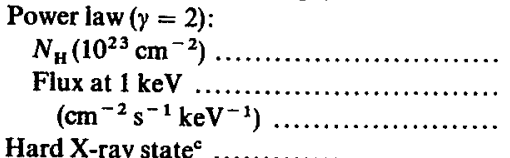 & $\cdots$ & $\begin{array}{l}1.32_{-0.20}^{+0.24} \\
0.11_{-0.06}^{+0.13}\end{array}$ & $\begin{array}{l}0.98_{-0.23}^{+0.25} \\
0.02_{-0.01}^{+0.03}\end{array}$ & $\begin{array}{l}1.18_{-0.15}^{+0.17} \\
0.05_{-0.02}^{+0.04}\end{array}$ \\
\hline Hard X-ray state ${ }^{c} \ldots \ldots \ldots \ldots \ldots \ldots \ldots \ldots$ & Low & Normal & Subluminous & $\cdots$ \\
\hline
\end{tabular}

aest fit assumes $k T=14.3 \mathrm{keV}$ (Kawai et al. 1988), errors are $68 \%$ confidence for $0.1 \mathrm{keV}<k T<100 \mathrm{keV}$.

bstimated by folding the combined PSPC spectrum through the HRI response.

c Mandrou et al. 1994.

Paradijs 1994) are labeled by name. Sources 2 and 3 are the HRI sources. Source 4 , located only $\sim 40^{\prime \prime}$ from source 2 , is a transient which appeared only during a single observation interval lasting $1400 \mathrm{~s}$. The small feature in the HRI image southwest of source 2 may also be due to this transient source. Table 2 gives coordinates for 1E 1740.7-2942 and sources 2, 3, 4 , and 5 , as well as $90 \%$ confidence error radii. The positions of sources 2 and 3 were determined in the same fashion as for $1 E$ 1740.7-2942. The position of source 4 was estimated from its offset from source 2 in the PSPC image and the position of source 5 was estimated from the PSPC image alone. We fitted the spectrum of source 2 in order to cross check or spectral analysis technique for $1 \mathrm{E} 1740.7-2942$. We find $k T=$ $0.77_{-0.28}^{+0.81} \mathrm{keV}, N_{\mathrm{H}}=4.7_{-1.6}^{+2.0} \times 10^{21} \mathrm{~cm}^{-2}$ and photon index $\gamma=3.7_{-1.2}^{+1.4}$ and $N_{\mathrm{H}}=6.4_{-2.3}^{+3.0} \times 10^{21} \mathrm{~cm}^{-2}$ for thermal bremsstrahlung and power law models, respectively. Both results are in good agreement with fits obtained using the XANADU/XSPEC (Shafer et al. 1990) package.

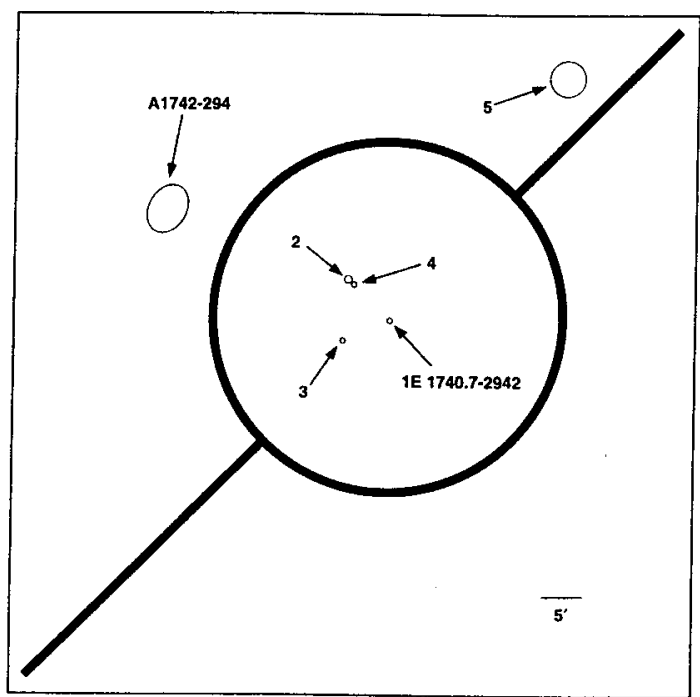

FIG. 5.-Diagram of the central region of the summed PSPC-I/II image. The actual image appears in Fig. 6. Sources 2 and 3 are the same as in the HRI. Among other detected sources are the well-known X-ray source A1742-294 (van Paradijs 1993), a transient, source 4, which appeared during a single $1400 \mathrm{~s}$ observation interval, and source 5, near the edge of the FOV. The circle and diagonal lines indicate PSPC window supports. North is up, and east is to the left.

\subsection{VLA Images}

As reported in Prince et al. (1991b), two weak sources, A and B, were detected in the Einstein/IPC error circle (see Fig. 1). Only source A (R.A. $=17^{\mathrm{h}} 43^{\mathrm{m}} 54^{\mathrm{s}} .75$, Decl. $=-29^{\circ} 44^{\prime} 42^{\prime \prime} \cdot 7$, epoch $\mathrm{J} 2000.0)$ is consistent with the more recent X-ray error circles from HEXE/TTM (Skinner et al. 1991) and ROSAT. It has a flux of $\sim 0.4 \mathrm{mJy}$ at $4.9 \mathrm{GHz}$ and is undetected at 1.5 GHz. Source B (R.A. $=17^{\mathrm{h}} 43^{\mathrm{m}} 54^{\mathrm{s}} \mathrm{s}$, Decl. $=-29^{\circ} 44^{\prime} 42^{\prime \prime} 0$, J2000), which lies outside of the new error circles, has a flux of $\sim 0.25 \mathrm{mJy}$ at $4.9 \mathrm{GHz}$ and $1.5 \mathrm{mJy}$ at $1.5 \mathrm{GHz}$ and is possibly extended. As part of their program of VLA monitoring of $1 \mathrm{E}$ 1740.7-2942, Mirabel et al. (1993) have analyzed the archival VLA data from these observations and presented coordinates and fluxes for sources A and B consistent with those given here.

\section{DISCUSSION}

\subsection{Association of the $X$-Ray and Radio Sources}

In Prince et al. (1991b), we suggested that the pointlike nature of source $A$ at $4.9 \mathrm{GHz}$ and the possible diffuse nature of source B made A the more likely counterpart to $1 \mathrm{E}$ 1740.7-2942. Shown in Figure 1 is the current X-ray error circle together with those from earlier measurements and the positions of A and B. As was the case with the TTM error circle (Skinner et al. 1991), the location of source A is consistent with that of the X-ray source while B lies outside of the error circle. Since our observations, Mirabel et al. (1992) have observed 1E 1740.7-2942 with the VLA in the C configuration which has increased sensitivity to low surface brightness objects. They found that source $\mathrm{A}$ is compact, time variable, and located at the center of double radio jets, the brighter of which is source B. They also estimated the probability that a random extragalactic source with a flux density of $0.4 \mathrm{mJy}$ would fall in the

TABLE 2

COORdinates (J2000) OF THE HRI AND PSPC SOURCES

\begin{tabular}{cccc}
\hline \hline Source & Right Ascension & Declination & $R_{\mathbf{9 0}}$ \\
\hline IE $1740.7-2942 \ldots \ldots$. & $17^{\mathrm{h}} \mathbf{4}^{\mathrm{m}} 54^{\mathbf{s}} .9$ & $-29^{\circ} 44^{\prime} 45^{\prime \prime} .3$ & $8^{\prime \prime} .5$ \\
$2 \ldots \ldots \ldots \ldots \ldots \ldots \ldots \ldots$. & 174417.7 & -293945.3 & 8.5 \\
$3 \ldots \ldots \ldots \ldots \ldots \ldots \ldots \ldots$. & 174421.5 & -294717.4 & 8.5 \\
$4 \ldots \ldots \ldots \ldots \ldots \ldots \ldots \ldots$. & 174415.4 & -294014.2 & 10 \\
$5 \ldots \ldots \ldots \ldots \ldots \ldots \ldots \ldots$ & 174216.3 & -291508.6 & $\sim 60$ \\
\hline
\end{tabular}





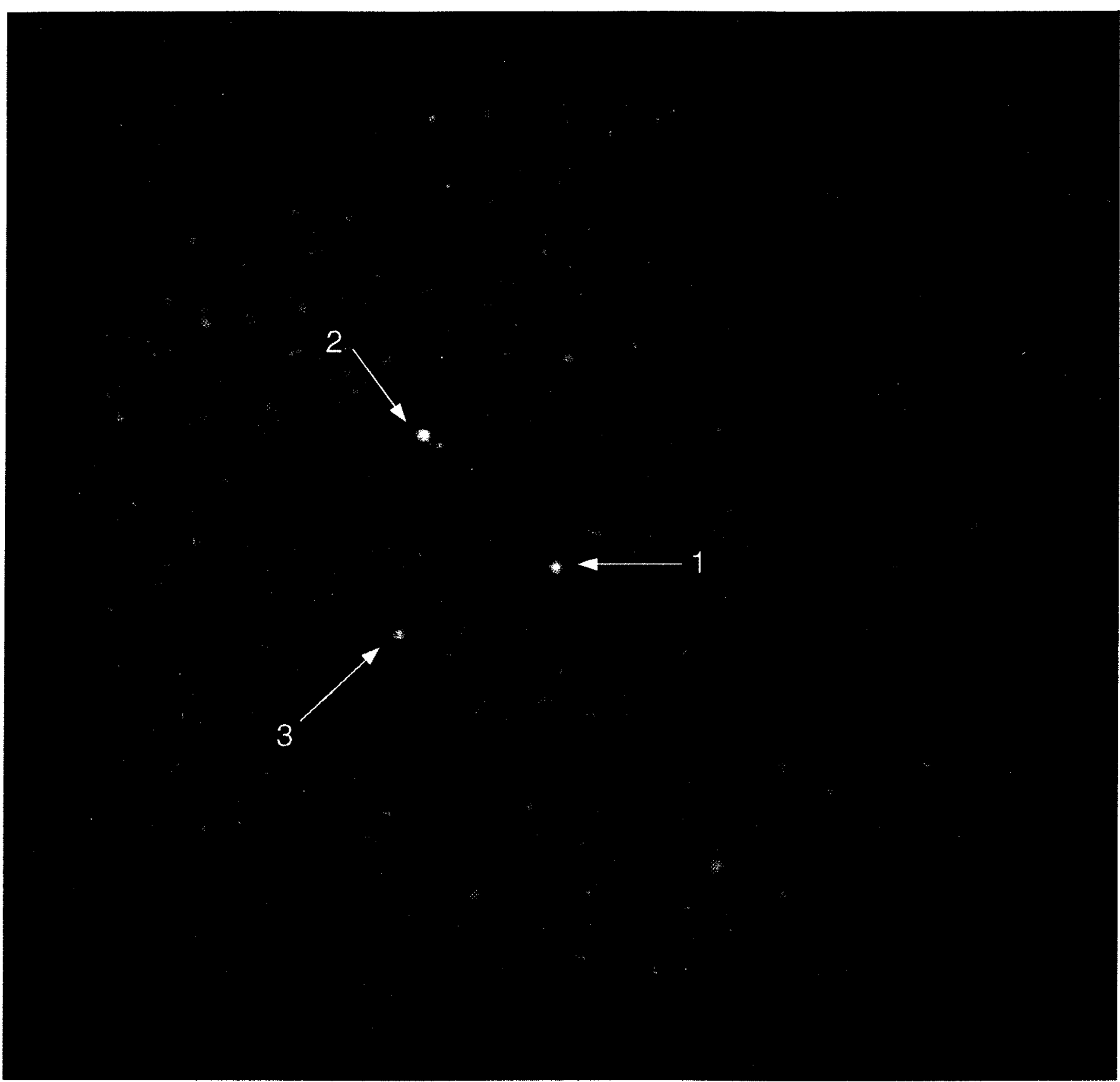

FIG. 3.-Gray-scale representation of the ROSAT HRI image of 1E 1740.7-2942. Sources are labeled as in Fig. 2. North is up, and east is to the left. HeindL, Prince, \& Grunsfeld (see 430, 829) 
A1742-294

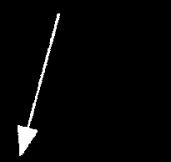

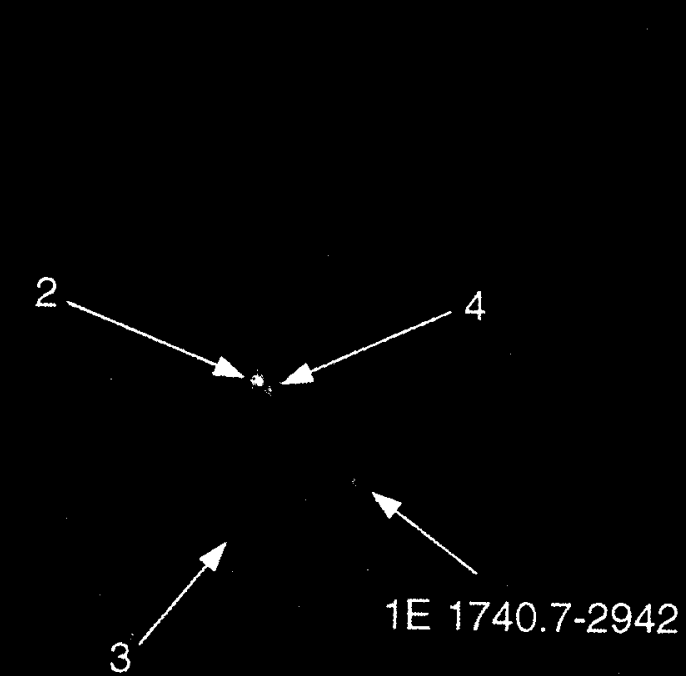

4

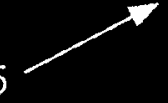

Fig. 6. -Gray-scale image of the center of the PSPC field of view. Sources are labeled as in Fig. 5. North is up, and east is to the left. Heindl, Prince, \& Grunsfeld (see 430, 830) 
